\title{
2 Von der Modellskizze zur Systemgestaltung
}

In diesem Kapitel wird ein handlungsorientiertes Modell zur Gestaltung in der Sozialwirtschaft ${ }^{1}$ präsentiert. Das Modell stellt eine Weiterentwicklung der Skizze eines systemischen Managementmodells für den Sozialbereich dar, dessen Grundzüge im Sammelband von Armin Wöhrle (2012) publiziert wurden (Bürgisser/ Buerkli/Stremlow/Kessler/Benz 2012: 231ff.). Es beschränkt sich dabei nicht nur auf das Management und die Leistungserbringung einer einzelnen sozialen Einrichtung, sondern nimmt auch den gesellschaftlichen Diskurs über soziale Probleme und dessen Bearbeitung durch politische Verantwortungsträger/-innen und weitere engagierte Akteure in den Blick. Im Weiteren fokussiert es das Zusammenspiel institutionsübergreifender sozialer Dienstleistungen in einem bestimmten Raum, wie zum Beispiel das Angebot an soziokulturellen, ambulanten und stationären Dienstleistungen im Bereich der Altenhilfe in einer Stadt oder einem Quartier.

\subsection{Der theoretische Bezugsrahmen}

Der theoretische Bezugsrahmen schliesst zunächst an Arbeiten von Stephan Müller und Jürgen Stremlow (2006) an, die auf der Basis der Systemtheorie ${ }^{2}$ zentrale Aspekte identifiziert haben, entlang derer sich das Funktionssystem Sozialwesen entwickelt oder ausdifferenziert. Dabei gehen sie von folgenden zwei Prämissen aus:

(1) Das Sozialwesen ist ein Funktionssystem (z.B. Baecker 1994, Maass 2009).

(2) Funktionssysteme entwickeln sich zum einen durch die Leitdifferenz von System und Umwelt (Luhmann 1987) und zum anderen entlang von Funktionen und Strukturen (Geser 1983).

Werden diese beiden zentralen Entwicklungsprinzipien miteinander in Verbindung gesetzt und auf das Funktionssystem Sozialwesen angewandt, entsteht eine

1 Die Begriffe «Sozialwirtschaft» und «Sozialwesen» werden hier gleichbedeutend verwendet.

2 Dementsprechend wird in der vorliegenden Publikation der Systembegriff in einem erweitert systemtheoretischen Verständnis verwendet. 
Tafel mit vier zentralen Entwicklungsbereichen, die für das Sozialwesen charakteristisch sind und entlang derer sich das Sozialwesen weiterentwickelt (Müller/Stremlow 2006).

\begin{tabular}{|l|l|l|}
\hline & $\begin{array}{l}\text { Problembezüge und profes- } \\
\text { sionelle Differenzierung so- } \\
\text { zialer Hilfe } \\
\text { (Funktion) }\end{array}$ & $\begin{array}{l}\text { Alimentierung und Struktu- } \\
\text { rierung Sozialer Hilfe } \\
\text { (Struktur) }\end{array}$ \\
\hline $\begin{array}{l}\text { Rahmenbedingungen und } \\
\text { Steuerung des Sozialwesens } \\
\text { (Umwelt) }\end{array}$ & $\begin{array}{l}\text { a) Soziale Probleme } \\
\text { (Problemartikulation) }\end{array}$ & $\begin{array}{l}\text { b) Sozialpolitische Steue- } \\
\text { rung }\end{array}$ \\
\hline $\begin{array}{l}\text { Leistungen und Strukturen } \\
\text { des Sozialwesens } \\
\text { (System) }\end{array}$ & $\begin{array}{l}\text { c) Professionelle Leistun- } \\
\text { gen } \\
\text { (Einrichtungen) }\end{array}$ & d) Versorgungssysteme \\
\hline
\end{tabular}

Abbildung 1: Die vier Entwicklungsbereiche des Sozialwesens Quelle: Müller/Stremlow (2006)

Soziale Probleme (Groenemeyer 1999) sind gesellschaftlich unerwünschte Entwicklungen, die den Bedarf nach sozialer Hilfe konstituieren. Dieser erste Entwicklungsbereich orientiert sich an der gesellschaftlichen Funktion des Sozialwesens.

Der Entwicklungsbereich 'sozialpolitische Steuerung' bezieht sich auf die politischen Interventions- und Steuerungsmassnahmen und gibt Auskunft über sozialpolitische Entwicklungen und deren Auswirkungen auf die einzelnen leistungserbringenden Einrichtungen und Versorgungssysteme in der Sozialwirtschaft. Der Entwicklungsbereich 'sozialpolitische Steuerung' wird ebenfalls zur Umwelt des Sozialwesens als Funktionssystem gezählt (politisches System).

Der Bereich 'professionelle Leistungen' beobachtet Entwicklungen und Veränderungen von Hilfsleistungen der Einrichtungen des Sozialwesens und die Strukturen dieser Einrichtungen. Professionelle Leistungen dieser Organisationen bezeichnen den Output des Sozialwesens.

Der vierte Entwicklungsbereich 'Versorgungssysteme' untersucht Entwicklungen respektive Veränderungen von Versorgungsstrukturen oder Versorgungsnetzwerken in einem definierten Raum. Dieser Entwicklungsbereich beschäftigt sich mit dem Zusammenspiel und den interinstitutionellen Kooperationsformen der verschiedenen Einrichtungen in der Sozialwirtschaft. 
Ausgehend von diesen vier systemtheoretisch begründeten Entwicklungsbereichen werden im folgenden Abschnitt Herausforderungen und Aufgaben für eine Gestaltung in der Sozialwirtschaft hergeleitet.

\subsection{Von den Entwicklungsbereichen zum Gestaltungsmodell}

Die Autoren dieses Buches schlagen vor, die vier Entwicklungsbereiche, die in der oben dargestellten Konzeption von Müller und Stremlow (2006) als zentrale Beobachtungsbereiche für die Entwicklungen des Funktionssystems Sozialwesen skizziert wurden, auf ein Gestaltungsmodell in der Sozialwirtschaft zu übertragen. In der folgenden Abbildung werden die Aufgaben und Rahmenbedingungen des Gestaltungsmodells kurz beschrieben.

\section{Diskurs soziale Probleme (a) \\ - Gesellschaftlicher Diskurs über soziale Probleme (Akteure, Medien, Themenusw.) \\ - Interessenvermittlung (Vernehmlas- sung, Interessenorganisation usw.)}

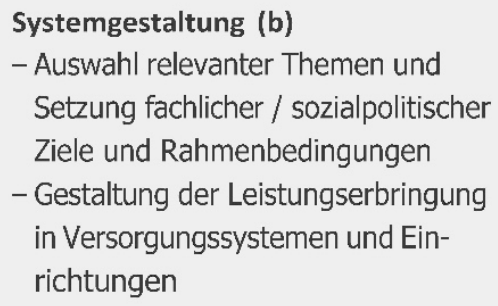

Versorgungssysteme (d)

Koordinationsfragen

Abbildung 2: Aufgaben / Rahmenbedingungen der Systemgestaltung Quelle: eigene Darstellung

Wie die Abbildung deutlich macht, umfasst das Modell zum einen handlungsorientierte Gestaltungsaufgaben. Die 'Systemgestaltung' ${ }^{3}$ (b) und das Management einer leistungserbringenden Organisation (c) stellen diese beiden zentralen Gestaltungsaufgaben dar, die von Akteuren unmittelbar ausgeübt werden. Zum anderen 
wird aufgezeigt, auf welche Rahmenbedingungen und Herausforderungen sich die verantwortlichen Akteure in ihrer Gestaltung ausrichten sollten. Der Diskurs über soziale Probleme (a) und die 'Versorgungssysteme' (d) stellen zentrale Anforderungen oder Rahmenbedingungen dar, mit denen Verantwortungstragende in der Sozialwirtschaft wie zum Beispiel Leitende von Einrichtungen oder Fachpersonen städtischer oder kantonaler Verwaltungen konfrontiert werden.

Viele Modelle und Betrachtungen im Sozialmanagementdiskurs legen den Schwerpunkt auf das Management einer einzelnen Einrichtung. Dies entspricht der Gestaltungsaufgabe (c), dem Management einer leistungserbringenden Organisation. Bernd Maelicke (2007: 923) zum Beispiel bezeichnet Sozialmanagement als «das Management von Betrieben und Unternehmen der Sozialwirtschaft in öffentlicher, privat-gemeinnütziger oder gewerblicher Trägerschaft». Dieses beinhalte «alle Managementfunktionen, die für die Führung und Leitung dieser Organisationen notwendig sind, so insbesondere Leitbild- und Konzeptionsentwicklung, Definition von Zielen und Aufgaben, Entwicklung der Aufbau- und Ablauforganisation, Personalmanagement, Führung und Zusammenarbeit, Innovationsmanagement, Sozialmarketing, Projektmanagement, Qualitätsmanagement, Controlling sowie andere Teildisziplinen des Managements» (ebd.). Im Gegensatz zum Management im Profit-Bereich berücksichtigt Sozialmanagement laut Maelicke (2007) zahlreiche Besonderheiten der Sozialbranche: die Spezifika von NonProfit-Organisationen (NPO), den sozialen Dienstleistungscharakter, die Einbeziehung von Ehrenamtlichen, die Mitgliederorientierung sowie die Probleme des Messens von Effektivität und Effizienz. Wöhrle erachtet die folgende Zusammenfassung von Klaus Grunwald (zit. in Wöhrle 2009: 149) als die zutreffendste: «(...), dass Sozialmanagement als wissenschaftlicher Begriff zu verstehen [ist], der sich mit der Leitung und Führung einer sozialen Einrichtung ${ }^{4}$ befasst und dabei unterschiedlichste Fragen der Organisationsgestaltung und Personalführung thematisiert».

Im Unterschied zu diesem weit verbreiteten Verständnis von Sozialmanagement plädieren die Autoren dieses Buches dafür, in ein entsprechendes Modell zusätzlich Fragen zur sozialpolitischen Systemgestaltung, zur Planung und Steuerung von Versorgungssystemen sowie den gesellschaftlichen Diskurs über soziale Probleme einzubeziehen.

4 In diesem Beitrag wird dem Begriff «soziale Einrichtung» Vorzug gegeben gegenüber jenem der NPO, da Letzterer einerseits recht willkürlich diverse Organisationen zusammenfasst, andererseits jedoch gewerbliche Anbieterinnen und Anbieter von sozialen Dienstleistungen nicht erfasst. Die Verwendung des Begriffes «NPO» ist darum nach Erachten der Autoren dieses Buches weder aus analytischer noch pragmatischer Sichtweise ergiebig (Begründung siehe Merchel 2006: 32ff.). 


\subsection{Element (a): Der Diskurs über soziale Probleme}

Wie weiter oben kurz beschrieben, beziehen sich soziale Probleme in der Regel auf bestimmte konkrete soziale Bedingungen, Strukturen oder Situationen, die als Störungen, Widersprüche oder Funktionsprobleme der Gesellschaft analysiert werden. Zur Definition gehört zudem die Wahrnehmung, Benennung oder soziale Konstruktion als Problem durch gesellschaftliche Akteure wie zum Beispiel Betroffene, Expertinnen und Experten, Professionelle, soziale Bewegungen oder Massenmedien. Als weiteres Bestimmungsmerkmal wird die Möglichkeit respektive Notwendigkeit formuliert - zum Beispiel durch das politische System -, die Situation zu verändern und Gegenmassnahmen zu entwickeln (Groenemeyer 1999: 15). Auch Friedrich Stallberg und Werner Springer (1983: 14) folgen dieser Auffassung und definieren soziale Probleme als «Phänomene, die 1) grössere Gruppen von Gesellschaftsangehörigen (bis hin zur Gesamtbevölkerung) in ihrer Lebenssituation beeinträchtigen, 2) öffentlich als veränderungsbedürftig definiert und 3) zum Gegenstand spezieller Programme und Massnahmen gemacht werden».

Dieser gesellschaftliche Diskurs respektive die Art und Weise, wie soziale Probleme öffentlich thematisiert werden, stellen diverse anspruchsvolle Anforderungen an das Management in der Sozialwirtschaft: zum einen an die politischen Verantwortungsträgerinnen und -träger, die in der Regel über die Ziele, den Vollzug und die Finanzierung von sozialpolitischen Massnahmen oder Hilfsprogrammen entscheiden und zum anderen an die Einrichtungen in der Sozialwirtschaft, die ihre Dienstleistungen und Angebote gegenüber verschiedensten gesellschaftlichen Anspruchsgruppen erbringen und zu legitimieren haben. Im Diskurs über soziale Probleme lassen sich diverse Aspekte unterscheiden, welche die Anforderungen in unterschiedlichster Weise prägen (Abbildung 3). 
Diskurs soziale Probleme (a)

Funktionsdiskurs: gesellschaft-

licher Diskurs darüber, was die

Aufgaben der Sozialwirtschaft

sind

Wertebezogenheit: Werte-

bezug / Legitimation sozialer

Dienstleistungen (Gerechtig-

keit, Solidarität, Chancengleich-

heit usw.)

Problemartikulation: Medien,

politisches System,

Wissenschaft, Betroffene und

ihre Vertretungen, ...

Interessenvermittlung: Formen

der Interessenvermittlung

(z.B.Vernehmlassungen)

zwischen den unterschiedlichen

Akteuren innerhalb des Staates
Systemgestaltung (b)

- Auswahl relevanter Themen

undSetzung fachlicher/

Sozial-

sozialpolitischer Ziele und

politische

Spannungs-

felder

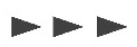

Rahmenbedingungen

- Gestaltung der Leistungserbringung in Versorgungssystemen und Einrichtungen

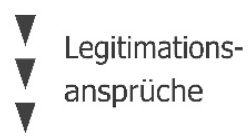

Leistungserbringende

Organisation (c)

Führung/Management

einer sozialen Einrichtung

Versorgungssysteme (d)

Koordinationsfragen

Abbildung 3: Soziale Probleme als Rahmenbedingung Quelle: eigene Darstellung

Im Rahmen eines Funktionsdiskurses wird eine gesellschaftliche Debatte darüber geführt, für welche sozialen Fragen, Bedingungen oder Situationen der Sozialstaat oder die Sozialwirtschaft in welcher Form zuständig sind. Diese Debatte ist häufig spannungs- und konfliktgeladen und wird von den unterschiedlichsten Positionen der beteiligten Akteure geprägt. In der Schweiz lassen sich seit Anfang der 1990er 
Jahre zunehmend polarisierende Auseinandersetzungen zu den Aufgaben des Sozialstaats und des Sozialwesens beobachten. Diese verstärkten öffentlichen Auseinandersetzungen haben dazu geführt, dass soziale Einrichtungen und politische Akteure ihre Tätigkeiten wesentlich stärker zu legitimieren haben.

Der Diskurs über soziale Probleme ist zudem von einer hohen Wertebezogenheit geprägt. In den Diskussionen wird oft auf soziale Gerechtigkeit, Chancengleichheit, Solidarität, Gemeinwohl oder Eigenverantwortung des Individuums rekurriert. Soziale Einrichtungen stehen vielfach im Spannungsfeld zwischen ideologischer Glaubwürdigkeit, Fachbezug und ökonomischer Realität; das heisst in einer anspruchsvollen Position zwischen Mission, Aufgabe und Markt. Die politischen Entscheidungsträgerinnen und -träger sind ebenfalls mit der Wertbezogenheit konfrontiert und haben bei der Formulierung sozialpolitischer Ziele und Massnahmen die wertbezogene Argumentation zu berücksichtigen.

Seit einiger Zeit lässt sich eine höhere mediale Präsenz sozialer Fragen beobachten. Zur ansteigenden Artikulation sozialer Probleme tragen sicher auch die vermehrte wissenschaftliche Forschung und die besser organisierten Vereinigungen und Vertretungen von Betroffenen bei.

Zudem existiert in den modernen Wohlfahrtstaaten ein ausdifferenziertes System der Interessenvermittlung zwischen den Bürgerinnen und Bürgern und dem politisch-administrativen System (Ladner 1991). Die Verschiedenheiten und die unterschiedlichen Positionen zwischen den Akteuren dieses Interessenvermittlungssystems - den Parteien, Interessenverbänden, sozialen Bewegungen, sozialen Einrichtungen und so weiter - prägen die Sozialpolitik massgeblich. Das daraus resultierende Konfliktpotenzial hat zur Folge, dass sozialpolitische Auseinandersetzungen auf nationaler Ebene in der Regel von sehr aufwändigen Prozessen der Konsensbeschaffung - wie dies insbesondere für die Schweiz zutrifft geprägt sind (Luhmann 1981). Die hohe funktionale Ausdifferenzierung der Interessenvermittlung geht zudem mit einer ausgeprägten Institutionalisierung und Formalisierung der Verfahren einher (z.B. Vernehmlassungsverfahren). Dies garantiert zwar eine hohe Transparenz, hat aber den Nachteil, dass diese Vorgänge aufwändig sind und entsprechend viel Zeit in Anspruch nehmen.

Diese Aspekte des gesellschaftlichen Diskurses geben Hinweise darauf, wie stark diese Auseinandersetzungen die Aufgaben der verantwortlichen Akteure im Rahmen der sozialpolitischen Entscheidungsfindung sowie die Positionierung und Legitimation sozialer Einrichtungen prägen. Dies stellt laufend neue Anforderungen und muss - unter anderem vom Management sozialer Einrichtungen - mitberücksichtigt werden. 


\subsection{Element (b): Systemgestaltung}

Unter der Systemgestaltung werden in diesem Buch die Aktivitäten von öffentlichen, privaten oder gewerblichen Trägerschaften verstanden, neue Dienstleistungen in der Sozialwirtschaft zu entwickeln oder bestehende Angebote durchzuführen und weiterzuentwickeln. Wie in der folgenden Abbildung gezeigt wird, können sich die Aktivitäten der Systemgestaltung auf eine einzelne Organisation oder auf Versorgungssysteme beziehen. Als ein Versorgungssystem wird die Gesamtheit aller Angebote oder Dienstleistungen, bezogen auf eine bestimmte und eingegrenzte Zielgruppe (z.B. Menschen mit Behinderung) innerhalb eines definierten und steuerungsrelevanten Sozialraums (z.B. Stadt, Quartier, Verbund von kommunalen Einheiten) bezeichnet, wobei sich die einzelnen Akteure als Teil eines von einer übergeordneten Instanz gesteuerten Systems erkennen. Damit wird auch zum Ausdruck gebracht, dass nach Erachten der Autoren die Summe aller Angebote allein noch nicht als Versorgungssystem bezeichnet werden kann, sondern erst dann, wenn sich die einzelnen Anbietenden als Systemakteure in wechselseitiger Bezogenheit mit andern Akteuren erkennen und sich diesem System ob freiwillig oder nicht - zugehörig fühlen.

Die Autoren haben die Aktivitäten der Systemgestaltung in vier zentrale Aufgabenpakete zusammengefasst. Sie werden in der folgenden Abbildung dargestellt. 


\section{Diskurs soziale Probleme (a) \\ - Gesellschaftlicher Diskurs über soziale Probleme (Akteure, Medien, Themen usw.) \\ - Interessensvermittlung (Vernehmlassungen, Interes- sensorganisationen usw.)}

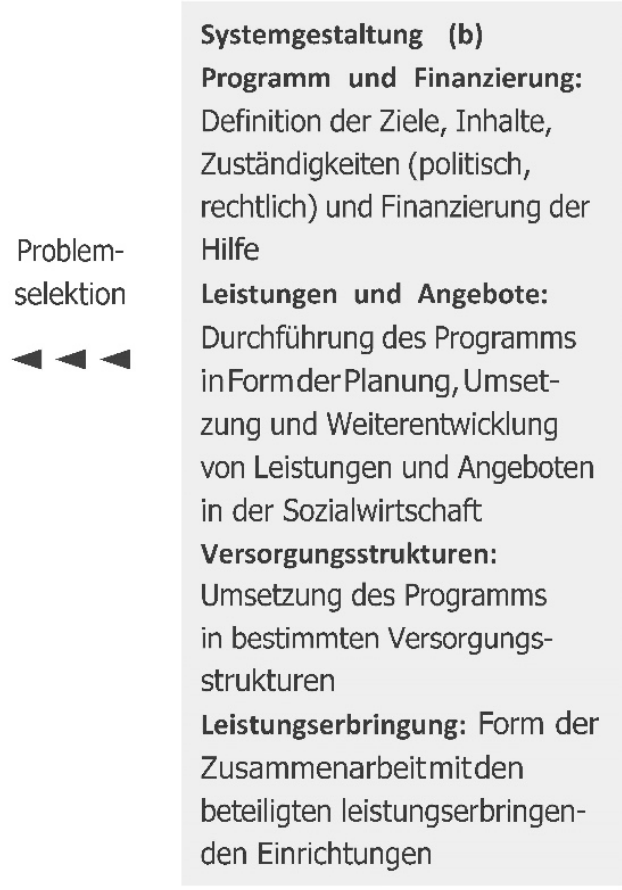

\section{Leistungs- vereinbarungen}

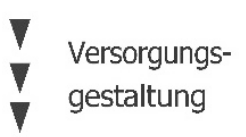

Versorgungssysteme (d)

Koordinationsfragen
Organisation (c)

Führung/Managementeiner sozialen Einrichtung

Abbildung 4: Aufgaben der Systemgestaltung Quelle: eigene Darstellung

Programm und Finanzierung: Bei der Entwicklung und Finanzierung von Programmen im Bereich der Sozialwirtschaft werden neue Dienstleistungen und Angebote geplant oder bestehende inhaltlich modifiziert, präzisiert oder Leistungen durch veränderte Ressourcenalimentierung priorisiert respektive zurückgestuft. Es 
werden Inhalte und Ziele festgelegt und die entsprechenden Mittel zugeordnet. Ist die öffentliche Hand Trägerin, kann bei der Programmierung und Finanzierung von sozialpolitischer Steuerung gesprochen werden. In diesem Fall definieren die politischen Verantwortungsträgerinnen und Verantwortungsträger das Programm und stellen dessen Finanzierung sicher. Dabei ist besonders zu berücksichtigen, dass beim Aufbau oder jeder Änderung eines Programms die politischen Verantwortlichkeiten auf den unterschiedlichen staatlichen Ebenen geklärt werden müssen. Dazu gehört sowohl die aktive strukturelle Klärung und Neuorganisation von Zuständigkeiten für die Programme - zum Beispiel im Rahmen der Neugestaltung des Finanzausgleichs und der Aufgabenteilung zwischen Bund und Kantonen in der Schweiz (NFA) - als auch die passive Anpassung von Programmen aufgrund von veränderten Zuständigkeitsregelungen zwischen den Gemeinden, den Kantonen (respektive Bundesländern) und dem Bund. In der Schweiz sind die Zuständigkeiten (rechtlich, finanziell und im Vollzug) oft auf verschiedenen staatlichen Ebenen und bei verschiedenen Akteuren angesiedelt. Dies gilt beispielsweise für die aktive Arbeitsmarktpolitik, die Pflege und Betreuung oder die Behindertenhilfe.

Bei der Gestaltung von Leistungen und Angeboten in der Sozialwirtschaft handelt es sich um die operative Umsetzung der Programme in Versorgungssystemen oder bei Einrichtungen durch die öffentliche Hand, die privaten oder die gewerblichen Trägerschaften. Im Rahmen der Dienstleistungsgestaltung legen die Verantwortungsträgerinnen und Verantwortungsträger fest, welche Leistungen im Rahmen eines Versorgungssystems erbracht werden sollen sowie welche Einrichtungen welche Leistungen in welcher Form zu erbringen haben und wie sie dafür entschädigt werden.

Versorgungsstrukturen: Analog zur Gestaltung von Dienstleistungen können die öffentlichen, privaten oder gewerblichen Trägerschaften auf die institutionellen Strukturen in der Sozialwirtschaft Einfluss nehmen, indem sie zum Beispiel die Grösse von Versorgungsregionen festlegen oder verändern, Vorgaben über Mindestgrössen bestimmter Einrichtungen machen, Schnittstellen und die Zusammenarbeit zwischen Einrichtungen definieren oder die Palette von sozialen Dienstleistungen oder die Angebotsdichte in einer Region festlegen. Die systematische strukturelle Gestaltung innerhalb von Versorgungsketten oder Versorgungssystemen gewinnt seit einigen Jahren zunehmend an Bedeutung; als Beispiele seien hier nur die Konzepte der «integrierten Versorgung» (Amelung/Sydow/Windeler 2009, Amelung/Meyer-Lutterloh/Schmid/Seiler/Weatherly 2008) oder der «Sozialraumorientierung» (Hinte/Litges/Springer 1999, Kessl/Reutlinger/Maurer/Frey 2011) genannt. 
Leistungserbringung: Seit etlichen Jahren sind in der Sozialwirtschaft Leistungsaufträge und Leistungsvereinbarungen als Steuerungsinstrumente üblich. Gemäss dem Konzept des NPM oder der wirkungsorientierten Verwaltungsführung sind Leistungsvereinbarungen wie folgt definiert: «Als Leistungsvereinbarungen werden sämtliche Aufträge, Kontrakte und Vereinbarungen bezeichnet, die Aufgaben, Kompetenzen und Verantwortung zwischen Politik, Verwaltungsführung, Verwaltungseinheiten und Dritten regeln», Schedler/Proeller 2006: 156). Die Leistungsvereinbarungen «konkretisieren die übergeordneten und operativen Ziele, die zu erbringenden Leistungen und die dafür zur Verfügung gestellten Mittel (Globalbudget). Verwaltungsintern kommt den Leistungsvereinbarungen die Funktion von Managementvereinbarungen zu, im Verhältnis zu Dritten sind es Verträge» (ebd.; siehe auch Ruflin 2006, Ruflin/Dvorak 2007). Leistungsvereinbarungen oder Leistungsverträge sind oft vergleichsweise komplizierte Regelwerke, namentlich was die Abrechnungs- und Abgeltungsmodalitäten der erbrachten Leistungen betrifft. Einrichtungen in der Sozialwirtschaft haben sich oft rasch an Veränderungen der Finanzierungs-, Abgeltungs- oder Abrechnungsmodalitäten anzupassen und sind diesbezüglich vor Herausforderungen gestellt. In jüngster Zeit entwickeln sich in der Zusammenarbeit zwischen der öffentlichen Hand und den Leistungserbringenden im Rahmen der New Public Governance neue Formen der Gestaltung ihrer Zusammenarbeit, wie zum Beispiel verstärkte Kooperationen in Netzwerken (Schubert 2015, Osborne 2010, Grunwald/Ross 2014).

\subsection{Element (c): Die leistungserbringende Organisation}

Der Sozialmanagementdiskurs konzentriert sich bislang auf das Management von leistungserbringenden Organisationen. $\mathrm{Zu}$ diesen Fragen liegen mit Abstand die meisten Publikationen, Konzepte, Handlungsanweisungen und -instrumente vor (Maelicke 2007, Simsa/Meyer/Badelt 2013, Merchel 2009, Wöhrle 2012, TabattHirschfeldt 2015). An dieser Stelle sollen jene Aspekte besprochen werden, die für eine leistungserbringende Organisation im Rahmen des Gestaltungsmodells für die Sozialwirtschaft besonders bedeutsam sind:

- Interessenvertretung im Diskurs sozialer Probleme;

- $\quad$ Legitimation gegenüber der sozialpolitischen Steuerung;

- Konkurrenz und Kooperation im Versorgungssystem;

- $\quad$ Führung und Management der Einrichtung, um die dem Organisationszweck entsprechende Leistung zu erbringen;

- Leistungserbringung im Kontakt und Austausch mit den leistungsbeziehenden Zielgruppen. 
Diskurs soziale Probleme (a)

- Gesellschaftlicher Diskurs über soziale Probleme (Akteure, Medien, Themen usw.)

- Interessensvermittlung (Vernehmlassungen, Interessensorganisationen usw.)

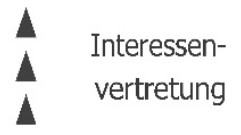

Leistungserbringende

Organisation (c)

- Interessenvertretung im Diskurs sozialer Probleme

- Legitimation gegenüber der leistungserbringenden Instanz und weiteren Anspruchsgruppen

- Konkurrenz und Kooperation im Versorgungssystem

- Führung und Management, um die dem Organisationszweck entsprechende Leistung zu erbringen

- Leistungserbringung im Kontakt und Austausch mit den leistungsbeziehenden Zielgruppen

\section{Systemgestaltung (b)}

- Auswahl relevanter Themen und Setzung fachlicher / sozialpolitischer Ziele und Rahmenbedingungen

- Gestaltung der Leistungserbringung in Versorgungssystemen und Einrichtungen

Qualitätsnachweis

Versorgungssysteme (d)

Koordinationsfragen
Abstimmung von Leistungen und Strukturen

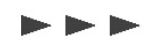

Abbildung 5: Aufgaben der leistungserbringenden Organisationen Quelle: eigene Darstellung 


\subsubsection{Interessenvertretung im Diskurs sozialer Probleme}

Wie im Abschnitt 2.3 zum Diskurs über soziale Probleme als Entwicklungstreiber diskutiert wurde, beziehen sich solche auf bestimmte reale soziale Bedingungen, Strukturen oder Situationen, die als Störungen, Widersprüche oder Funktionsprobleme der Gesellschaft analysiert werden. Es wird eine gesellschaftliche Debatte darüber geführt, für welche sozialen Aufgaben der Sozialstaat und die Sozialwirtschaft in welcher Form zuständig sind. Daraus können neue oder modifizierte Aufträge bezüglich der sozialpolitischen Gestaltung resultieren, die anschliessend an leistungserbringende Organisationen übertragen werden können. Aus dieser Konstellation ergeben sich anspruchsvolle Aufgaben für das Management der jeweiligen Organisation. Wie soll sich eine soziale Einrichtung gegenüber diesem Diskurs verhalten und positionieren? Sie kann sich beispielsweise als interessierte Zuschauerin passiv verhalten. Sie kann aber auch eine aktive Rolle übernehmen, sich direkt am Diskurs beteiligen und auf diesem Weg versuchen, Einfluss zu nehmen oder sie kann indirekt über Netzwerke versuchen, ihren Einfluss geltend zu machen.

Welche Option für eine soziale Einrichtung sinnvoll erscheint, ist von ihrem Selbstverständnis, ihrer Identität und ihrem Auftrag abhängig. In Anbetracht dessen, dass sich soziale Einrichtungen - insbesondere im Fall einer privaten Trägerschaft - ihre Bestimmung und ihre Ziele oftmals selber geben, oder aber den Auftrag von einer staatlichen Stelle im Rahmen eines Leistungsauftrags übernommen haben (Herzka 2013: 19), wird deutlich, wie eng die Positionierung im Diskurs sozialer Probleme mit dem Auftrag und der Mission einhergeht. Je nach Mission der Organisation können dabei Ziele im Zusammenhang mit politischen Kampagnen oder auch die Interessen der Klientel im gesellschaftlichen Diskurs zusätzlich zu den operativen Dienstleistungszielen ihren Stellenwert haben.

\subsubsection{Legitimation}

Die Systemgestaltung ist im Wesentlichen durch zwei Prozesse geprägt: 1) das Fällen von gesellschaftlich verbindlichen Entscheiden (Parlament, politische Parteien) und 2) die Umsetzung der getroffenen Entscheide durch Regierung und Verwaltung (Druwe 1995). Das Management der leistungserbringenden Organisation hat beide Prozesse im Auge zu behalten. In der Sozialwirtschaft werden Leistungen heute in der Regel mittels Leistungsvereinbarungen geregelt, die zwischen der sozialpolitischen Gestaltungsebene, meistens eine zuständige Amtsstelle, und der operativen Umsetzungsebene, meistens eine staatlich oder privat getragene soziale Organisation, abgeschlossen werden. Daraus ergibt sich eine gegenseitige Abhängigkeit, die es durch das Management der leistungserbringenden Organisation zu 
berücksichtigen gilt. Alle beteiligten Akteure stehen unter einem Legitimationsdruck. Aus der Sicht der sozialpolitischen Gestaltung und Steuerung haben die leistungserbringenden Organisationen einen sozialpolitischen Auftrag zu erfüllen und ein bestimmtes Versorgungsniveau zu sichern. Dabei soll mit den vorhandenen Ressourcen das bestmögliche Ergebnis erzielt werden (Meinhold/Matul 2011: 79ff.). Diese Zielsetzung wird von den politisch Verantwortlichen eingefordert und muss je nach politischer Opportunität belegt und dokumentiert werden können. Dies führt zusätzlich zu einem Legitimationsdruck auf die leistungserbringenden Organisationen und diese Legitimationsanforderung ist zu gestalten.

Um die Tätigkeit einer leistungserbringenden Organisation zu legitimieren, sind vier wesentliche Erfordernisse zu erfüllen:

(1) Zuerst sind ein systematisches Monitoring, ${ }^{5}$ ein damit einhergehendes Berichtswesen sowie eine regelmässige Kommunikation zwischen den Vertragspartnerinnen und -partnern notwendig.

(2) Ein zweites Erfordernis stellt die Sicherung und Entwicklung der Qualität dar. Gemäss Marianne Meinhold und Christian Matul (2011), die ein breit gefasstes und mehrdimensionales Qualitätsverständnis vertreten, müssen dabei verschiedene, sich möglicherweise auch widersprechende Logiken der politischen Entscheidungsebene, der Verwaltung, des Managements einer Einrichtung, der Fachkräfte und der leistungsbeziehenden Personen berücksichtigt werden. ${ }^{6}$ Dieses Verständnis von Qualität setzt voraus, dass die Vertragsparteien eine gemeinsame Grundlage und Sichtweise gegenüber der Qualität im Rahmen der Leistungserbringung erarbeitet haben. Dies kann auf der organisationalen Ebene durch ein bestimmtes Qualitätsmanagement-System wie $\mathrm{EFQM}^{7}$ oder andere Systeme umgesetzt werden.

(3) Das dritte Erfordernis, die Legitimation der leistungserbringenden Organisation gegenüber der leistungsbestellenden Instanz zu gewährleisten, ist eine angemessene Non-Profit-Governance. Ein organisationales Governance-System meint die Gesamtheit aller steuerungswirksamen Strukturen in einer Organisation, welche die Erfüllung von Rechenschaftspflichten und den Schutz von Interessen der Anspruchsgruppen sicherstellt (Maier/Meyer 2013: 492). Gesetzliche Vorgaben wie zum Beispiel das Vereins- oder Stiftungsrecht geben

5 Monitoring bezeichnet gemäss Joachim Hoehn (2013: 675) einen systematischen Prozess der Erfassung, Beobachtung und Überwachung von sozialen, betrieblichen und unter anderem Entwicklungen anhand mehrdimensionaler Beobachtungssysteme auf Basis definierter Indikatoren.

6 Weitere Ausführungen zur Qualitätssicherung sind im Kapitel 3 dargelegt.

7 Das EFQM (für: European Foundation of Quality Management) ist ein in Europa verwendeter Rahmen für Managementmodelle von Organisationen. Nähere Informationen dazu finden sich unter http://www.efqm.ch/. 
hier Minimalanforderungen vor, wobei die konkrete Ausgestaltung der Governance über die gesetzlichen Mindestanforderungen hinausgehen kann und dies aufgrund der Legitimationsanforderung an die Governance auch tun sollte.

(4) Als viertes Erfordernis ist die Pflege von persönlichen Beziehungen und Kontakten zu den Vertreterinnen und Vertretern der leistungsbestellenden Instanzen zu nennen. Letztlich arbeiten in diesem Kontext Menschen zusammen, die auf gegenseitige Akzeptanz und Vertrauen angewiesen sind. Vertrauen ist das wichtigste Kapital einer sozialen Organisation. Dieses kann nicht nur durch Managementinstrumente aufgebaut und erhalten werden. Gute, verlässliche persönliche Beziehungen und ein integres Verhalten der Führungspersonen und der Mitarbeitenden legen das Fundament für eine Legitimation. In ihren Thesen zum wohlfahrtstaatlichen Kontraktmanagement postuliert Regula Ruflin als eine ihrer Thesen, dass bestehende Beziehungen, Vertrauen und Stabilität das Zustandekommen von Leistungsverträgen bestimmen sollten und nicht Marktelemente wie Preis, Quantität und Qualität (Ruflin 2006: 92).

\subsubsection{Konkurrenz und Kooperation im Versorgungssystem}

Versorgungssysteme können auch als eine Art Märkte gesehen werden, in denen Anbietende und Nachfragende von sozialen Dienstleistungen zusammentreffen. Im Gegensatz zu den marktwirtschaftlichen Mechanismen werden in der Sozialwirtschaft jedoch die Bedingungen und Formen des Austauschs von Dienstleistungen (Qualität, Preis, Menge usw.) nicht von den leistungsbeziehenden Personen und den sozialen Einrichtungen alleine definiert. Die Rahmenbedingungen, unter denen eine Dienstleistung entwickelt und angeboten werden kann, werden oftmals massgeblich durch politische Instanzen mitgestaltet. Im Sozialmanagement wird in diesem Zusammenhang von nicht schlüssigen Tauschbeziehungen (Merchel 2009: 78) gesprochen. Obwohl die klassischen Marktmechanismen hier kaum von Bedeutung sind, haben sich in der Sozialwirtschaft oft regionale Versorgungssysteme gebildet, in denen soziale Einrichtungen agieren und dabei in Konkurrenz treten oder kooperieren.

Aus der Sicht der einzelnen sozialen Einrichtung, aber auch für die sozialpolitische Steuerung, ist es dabei wichtig, diesen spezifischen «Markt» zu verstehen.

Gilt es, ein Versorgungssystem zu gestalten und weiterzuentwickeln, ist die Analyse der Angebotssituation ein wichtiger Schritt. Verfolgen mehrere soziale Einrichtungen dasselbe Ziel, entsteht Konkurrenz beziehungsweise Wettbewerb. Den steuerungsverantwortlichen Instanzen bietet sich die Möglichkeit, Angebote, Preise und Leistungen zu vergleichen. Renate Buber (2013: 231) weist in diesem Kontext darauf hin, dass Wettbewerb unter NPO als Leistungsanbietende häufig 
auf der Qualität der Dienstleistungen, der Fähigkeit, einzelne Bedürfnisse zu erfüllen und auf der Reputation beruht. Aus Sicht der Nachfragenden von NPOLeistungen besteht die Konkurrenz bei der Auftragsbeschaffung und der Finanzierung sowie beim Fundraising. Angesichts des Spardrucks der öffentlichen Hand wird zusätzlich die Preisgestaltung für einzelne Dienstleistungen immer wichtiger. «Zunehmender Wettbewerb übt einen Druck in Richtung Auftrags- und Markzentrierung aus, die Mission der NPO tritt damit in den Hintergrund. Prekär wird in diesem Zusammenhang insbesondere jene Funktion von NPO, die über die Produktion von sozialen Dienstleistungen hinausgeht: die Vertretung von (schwachen) Interessen, die sonst nicht vertreten würden (advocacy, voice)» (Meyer 2007: 66). Auf die Frage, inwieweit Versorgungssysteme in der Sozialwirtschaft durch Kooperation gestaltet werden können und sollen, wird im Abschnitt 3.2 eingegangen.

\subsubsection{Führung und Management in der Organisation}

Wie zu Beginn dieses Kapitels erwähnt, steht das Management von leistungserbringenden Organisationen bislang im Fokus des Sozialmanagements. Es existiert dazu eine reichhaltige Fachliteratur.

Joachim Merchel (2009: 129) beschreibt Sozialmanagement als mehrdimensionale Steuerung. Nachfolgende Ausführungen zu zentralen Managementaspekten orientieren sich am Modell von Merchel und werden mit eigenen Überlegungen der Autoren ergänzt:

- Fachlich: Eine rein managementbezogene Perspektive mit einseitig ökonomisch inspirierten Managementmethoden erscheint gegenüber den Anforderungen des Sozialmanagements unangemessen. Es gilt der Sachzieldominanz in sozialen Einrichtungen Rechnung zu tragen und qualitativ hochwertige Leistungen zu erbringen. Merchel (2009) plädiert dafür, dass die jeweilige Eigenlogik von Sozialer Arbeit und Management zum Tragen kommt und daraus entstehende Spannungen nicht in eine einseitige Richtung aufgelöst werden. Insbesondere beim Qualitätsmanagement wird die Verknüpfung von Management mit fachlichen Ansprüchen deutlich.

- Organisationsbezogen: Innerorganisationale Strukturen wie die Aufbau- und die Ablauforganisation, Prozesse, physische Mittel, Funktionen und Organe sind optimal zu gestalten, damit die soziale Einrichtung ihre dem Organisationszweck entsprechende Leistung erbringen kann. Dazu gehört auch die Gestaltung der nach aussen gerichteten Bezüge, beispielsweise gegenüber der sozialpolitischen Steuerung oder gegenüber anderen Akteuren. 
- Personen- und gruppenbezogen: Personenbezogene Faktoren haben bei der Erbringung von sozialen Dienstleistungen eine elementare Bedeutung. Fachlich qualifiziertes, motiviertes Personal bildet daher eine Schlüsselressource. Ein angemessener Umgang mit den Mitarbeitenden, eine sorgfältige Kommunikation und die Berücksichtigung von Team- und Gruppenkonstellationen, formellen und informellen Dynamiken, die Moderation von Spannung und Konflikten und dergleichen gehören zu den Steuerungsanforderungen.

- Betriebswirtschaftlich: Soziale Einrichtungen sind heute anspruchsvollen betriebswirtschaftlichen Rahmenbedingungen ausgesetzt und müssen ihre personellen und finanziellen Ressourcen optimal einsetzen. Die sozialpolitische Steuerung stellt finanzielle Ressourcen auf der Basis von Leistungsvereinbarungen zur Verfügung. Pauschale Abgeltungs- und Subventionsmodelle im Sinn von Objektfinanzierungen wurden durch unterschiedliche Modelle von Subjektfinanzierungen ergänzt und teilweise abgelöst. Bei öffentlich finanzierten Einrichtungen, aber auch bei Einrichtungen, die sich ganz oder teilweise über Spenden, Legate oder Stiftungen finanzieren, wird eine transparente, zweckgerichtete Mittelverwendung verlangt. Betriebswirtschaftliche Steuerung im Sozialmanagement erfordert daher eine transparente Darstellung von Geschäftsvorgängen mittels Bilanzierung, Budgetierung, Kostenund Leistungsrechnung und Controlling. Neben den finanziellen Kennzahlen verlangen Behörden und Öffentlichkeit zunehmend auch leistungszielbezogene Kennzahlen.

- Umgang mit rechtlichen Steuerungsmodalitäten: Die gesetzlichen und rechtlichen Grundlagen des Sozialstaats zur Absicherung von sozialen Risiken bilden einen wichtigen Bezugsrahmen für soziale Einrichtungen. Leistungsverträge zwischen der öffentlichen Hand und sozialen Einrichtungen basieren auf jeweils auftragsbezogenen, spezifischen rechtlichen Grundlagen. Neben diesen - durch die Sachziele und den Organisationszweck gegebenen - rechtlichen Rahmenbedingungen ist oft eine grosse Zahl weiterer Gesetze und rechtlicher Vorgaben zu berücksichtigen.

- Reflexion und Gestaltung der Aussenbezüge: Die Leistungen von sozialen Einrichtungen stehen in einer Wechselwirkung zu gesellschaftlichen und sozialpolitischen Entwicklungen. Merchel (2009: 133) betont daher: «Es gehört zum integralen Bestandteil des Managements von Einrichtungen der Sozialen Arbeit, die Veränderungen in ihrer gesellschaftlichen, ökonomischen und politischen Umwelt sehr genau wahrzunehmen und zu analysieren, um strategische Entscheidungen in ihrer Institution treffen zu können». Diese Umweltentwicklungen können Einfluss auf politische Entscheidungen und die Leistungen haben, die durch die öffentliche Hand in der Sozialwirtschaft finanziert werden. Aber auch die Sachziele der sozialen Organisation können 
sich durch gesellschaftliche Entwicklungen verschieben. Neue, ungelöste Probleme in der Gesellschaft, benachteiligte Bevölkerungsgruppen und so weiter können zur Entwicklung neuer Angebote führen.

\subsubsection{Leistungserbringung im Kontakt mit den Zielgruppen}

Das vorliegende Modell erfasst einerseits Prozesse, die auf der Makroebene anzusiedeln sind, wie beispielsweise den gesellschaftlichen Diskurs zu sozialen Problemen und dessen Auswirkungen auf die Gesetzgebung und die Vorgaben an die sozialpolitische Steuerung. Gleichzeitig hat die Mesoebene der leistungserbringenden sozialen Organisationen einen hohen Stellenwert. Weniger zum Tragen kommt auf den ersten Blick die Mikroebene mit der direkten Interaktion zwischen den Personen, die beispielsweise als Klientinnen und Klienten Leistungen beziehen, und den Fachpersonen, die diesen Leistungsbezug sicherstellen. Diese Interaktionen haben jedoch entscheidende Bedeutung, damit soziale Einrichtungen ihre Ziele erreichen können. So haben bereits Bernhard Badura und Peter Gross (1976) den Charakter und die Eigenlogik von personenbezogener Dienstleistungstätigkeit auch in der Sozialen Arbeit betont. Produktion und Bezug der Leistung fallen an einem Ort zusammen (uno-actu-Prinzip). Der Kern personenbezogener professioneller Dienstleistungen besteht aus dem persönlichen Kontakt zwischen den Professionellen als Dienstleistungsanbietende und den Klientinnen und Klienten als Dienstleistungsbeziehende. Die Qualität der Dienstleistung oder die Ergebnisse eines Beratungsprozesses sind in hohem Mass von der Interaktion der beteiligten Akteure beeinflusst. Sie werden in einer sogenannten Ko-Produktion erzielt. Neben personenbezogenen sozialen Dienstleistungen (Beratung, Betreuung, Bildung, Erziehung, Pflege) können auch haushaltsbezogene soziale Dienstleistungen (Zubereitung von Mahlzeiten, Unterhalt der Wohnungseinrichtung usw.) erbracht werden.

Auch Claus Reis und Lutz Wende (2010: 78) betonen, dass die Betrachtung personenbezogener Dienstleistungen die Probleme der Organisation und Steuerung einbeziehen muss. Politisch gesetzte Ziele wie zum Beispiel die Integration einer langzeitarbeitslosen Person in den regulären Arbeitsmarkt können nur erreicht werden, wenn eine funktionierende Ko-Produktion zwischen Professionellen und Klientinnen und Klienten stattfindet. Dies beinhaltet auch die Partizipation der Letzteren im Prozess.

Bei der Systemgestaltung, die jeweils auf bestimmte sozialpolitische Ziele ausgerichtet ist, hat die beschriebene Ko-Produktion auf der Mikroebene eine grosse Bedeutung. Hier entscheidet sich, ob die gewünschten Wirkungen erzielt werden können. Dabei sind der Einfluss des Managements von Einrichtungen und derjenige der sozialpolitischen Steuerungsebene begrenzt. Entscheidend sind die 
Professionalität, die fachliche Qualifizierung, die persönliche Eignung und die Motivation der Mitarbeitenden in den sozialen Einrichtungen.

\subsection{Element (d): Koordination in Versorgungssystemen}

Unter einem Versorgungssystem wird ein interorganisationales Netzwerk von Einrichtungen verstanden (Amelung/Sydow/Windeler 2009). Gemäss Müller und Stremlow (2006) ist die Versorgungsqualität - aber auch der Zugang zur oder die Effektivität und die Effizienz von Leistungen - davon abhängig, wie angemessen die Aufgabenteilung zwischen den einzelnen Einrichtungen organisiert ist und wie zweckmässig die Aufteilung zwischen zentralen und dezentralen Angeboten geschieht. Eine Region kann mit Angeboten in einem bestimmten Bereich noch so gut versorgt sein - die investierten Ressourcen erzeugen keine/n optimale/n Versorgungszugang oder -qualität, wenn die einzelnen Angebote nicht aufeinander abgestimmt sind. Dies ist der Fall, wenn zum Beispiel Doppelspurigkeiten zwischen Angeboten existieren, Leistungen erbracht werden, die nicht ins Gesamtsystem eingebunden sind, Drehtüreffekte respektive die Rückkehr der Situation in den Anfangszustand festgestellt werden können oder keine angemessene Kooperation zwischen den Einrichtungen stattfindet. Müller und Stremlow (2006) vertreten die Auffassung, dass der Output einer einzelnen Einrichtung im Sinn eines Outcomes erst dann wirklich nutzbar sei, wenn er auf der Ebene des Versorgungssystems übersetzt werde. Im Gesundheitswesen gewinnen Modelle der integrierten Versorgung (Amelung/Sydow/Windeler 2009, Amelung/Meyer-Lutterloh/ Schmid/Seiler/Weatherly 2008), die vergleichbare Ziele anstreben, zunehmend an Bedeutung. Auch Wolf Rainer Wendt (2009) kommt zum Schluss, dass in der Sozialen Hilfe im Einzelfall komplexe Lösungen gefragt sind, die nicht von einer Leistungsanbieterin oder einem Leistungsanbieter bereitgestellt werden können. Aus seiner Sicht bedarf es deshalb vermittelnder Strukturen und der Bereitschaft von Diensten und Einrichtungen, verbindliche Absprachen zu treffen und einzuhalten. Versorgungssysteme sind häufig zugleich von Wettbewerb und Kooperation der teilhabenden Einrichtungen geprägt (Amelung/Sydow/Windeler 2009). Sie stellen an die Koordination oder Steuerung spezifische Anforderungen, die im Folgenden kurz beschrieben werden. Vier Aspekte scheinen den Autoren des vorliegenden Buches besonders relevant zu sein (Abbildung 6):

- $\quad$ Ausstattung und Dichte der Angebote (Basisinformationen);

- Angebotsabstimmung und -koordination in der Versorgungskette;

- räumliche Gestaltung;

- $\quad$ Lenkung der Leistungsbezügerinnen und Leistungsbezüger. 
Diskurs soziale Probleme (a)

- Gesellschaftlicher Diskurs

über soziale Probleme

(Akteure, Medien, Themen usw.)

- Interessensvermittlung (Vernehmlassungen, Interessensorganisationen usw.)

\section{Leistungserbringende \\ Organisation (c) \\ Führung/Managementeiner \\ sozialen Einrichtung}

\author{
Systemgestaltung (b) \\ - Auswahl relevanter Themen \\ und Setzung fachlicher/ \\ sozialpolitischer Ziele und \\ Rahmenbedingungen \\ - Gestaltung der Leistungs- \\ erbringung in Versorgungs- \\ systemen und Einrichtungen
}

\section{Anforderungenandie \\ Systemgestaltung}

Abbildung 6: Koordinationsfragen in Versorgungssystemen Quelle: eigene Darstellung 


\subsubsection{Ausstattung und Dichte der Angebote}

Versorgungssysteme zeichnen sich zunächst durch eine bestimmte Ausstattung mit Angeboten oder Dienstleistungen aus. Welche Angebotspalette in einem Versorgungssystem zur Verfügung steht, ist aus der Sicht von Planung und Steuerung eine Basisinformation. Daneben sind auch die Dichte oder der Umfang der Angebote, die in der Regel zu Bevölkerungszahlen, Risiko- oder Zielgruppen in Beziehung gesetzt werden, ein weiteres grundlegendes Merkmal von Versorgungsnetzwerken. Häufig kann ein Quervergleich der Angebotspalette und -dichte von Versorgungssystemen in vergleichbaren Aufgabengebieten (z.B. Langzeitpflege oder Angebote für Menschen mit Behinderung) wichtige Hinweise für die Planung und Weiterentwicklung geben. Ausstattung und Dichte der Angebote sind demzufolge zentrale Informationen für die weiterführende Gestaltung und Entwicklung von Versorgungssystemen oder -netzwerken.

\subsubsection{Angebotskoordination in der Versorgungskette}

Angesichts der zunehmenden Angebotsentwicklung und Angebotsspezialisierung, die in der Sozialen Arbeit und an den Schnittstellen zum Gesundheits- oder Bildungssystem zu verzeichnen ist, gewinnt die Koordination der Angebote entlang der Versorgungskette an Bedeutung (Wendt 2009). Die Entwicklung von Konzepten integrierter Versorgung oder Versorgungsmodelle der letzten Jahre macht dies deutlich. Ziel dieser Bemühungen ist es, Doppelspurigkeiten, Drehtüreffekte oder Angebotslücken in Versorgungsabläufen zu erkennen und die Kooperation der einzelnen Dienste oder Einrichtungen miteinander zu erhöhen. In jüngster Zeit wurden dazu auch handlungsorientierte Modelle entwickelt wie zum Beispiel das «Quadranten-Modell der Integration» oder das Modell der sogenannten «polykoordinierten Versorgung» (Pfaff/Kowalski/Ommen 2009), um mögliche Ausschlussprozesse in Versorgungssystemen zu minimieren. Angebotskoordination ist damit ein weiterer zentraler Aspekt der Gestaltung und Optimierung in Versorgungssystemen in der Sozialwirtschaft. In der Schweiz schaffen die föderalistischen Strukturen und die historisch gewachsene Angebots- und Einrichtungslandschaft mit unterschiedlichen Finanzierungsquellen eher schwierige Ausgangsbedingungen für eine am Beratungs- oder Betreuungsprozess orientierte Angebotsabstimmung. Integrierte Versorgungsmodelle (Amelung/Meyer-Lutterloh/Schmid/ Seiler/Weatherly 2008), die sich konsequent an den Kernprozessen und Kerndienstleistungen orientieren - zum Beispiel im Bereich Betreuung und Pflege stehen auch in der Schweiz zur Diskussion. 


\subsubsection{Räumliche Gestaltung von Versorgungssystemen}

Die räumliche Gestaltung von Versorgungssystemen befasst sich mit der Frage, an welchen Standorten innerhalb einer Versorgungsregion welche Angebote zur Verfügung gestellt werden können oder sollen. Es geht um Fragen der geeigneten Grösse von Einzugsgebieten, Fragen der Erreichbarkeit der Angebote aus der Sicht der Klientinnen und Klienten oder Betroffenen oder um solche der Konzentration und Zentralisierung von hoch spezialisierten Angeboten im Spannungsfeld zwischen guter Erreichbarkeit und notwendiger Organisationsgrösse für einen spezialisierten Kompetenzaufbau. Seit dem Ende der 1970er Jahre hat sich innerhalb der Sozialen Arbeit eine hohe Spezialisierungs- und Bürokratisierungsdynamik entwickelt, die seit längerer Zeit kritisiert wurde (Bronke/Wenzel 1980) und bis heute noch wird. Als Gegenmodell werden Konzepte zur sozialraumorientierten Angebotsgestaltung entwickelt (Hinte/Litges/Springer 1999, Schubert 2017), welche dezentrale polyvalente Dienste favorisieren, die verschiedenen Angebote unter einem Dach zusammenfassen und für Klientinnen und Klienten gut erreichbar in deren Lebensräumen angesiedelt sind. In der Frage der räumlichen Gestaltung von Versorgungssystemen bleiben die Spannungsfelder zwischen dezentralen und zentralen sowie zwischen polyvalenten und spezialisierten Organisationsformen immanent. Auf die in diesem Abschnitt referierten Fragen existieren keine allgemein gültigen Antworten und es sollte für jedes spezifische Versorgungsnetz analysiert und entschieden werden, welche Positionierung im Raum als adäquat bezeichnet werden kann. In der Schweiz sind Fragen nach der adäquaten Grösse und den Standorten sozialer Einrichtungen angesichts vieler kleiner Einzugsgebiete und politischer Zuständigkeitsbereiche besonders bedeutend.

\subsubsection{Nutzerbezogene Lenkung}

Die Lenkung der Leistungsbeziehenden ist auf der Ebene der Nutzerinnen und Nutzer von sozialen Einrichtungen das Pendant zur Angebotskoordination entlang der Versorgungskette auf der Strukturebene. Angebote können ihre volle Leistungsfähigkeit nur entfalten, wenn ihre Ziele und Massnahmen auf die sozialen Probleme, die Risiken und / oder die Bedürfnisse der Zielgruppen abgestimmt sind. Verschiedene aktuelle Studien zu Arbeitsintegrationsprogrammen für Sozialhilfebezügerinnen und Sozialhilfebezüger in der Schweiz weisen auf eine ungenügende Passung der Integrationsprogramme und der Zielgruppen hin (Aeppli/ Ragni 2009, Schallberger/Wyer 2009). Auch in anderen Bereichen wie der Pflege und Fürsorge oder der Betreuung von Menschen mit Behinderung wird eine teilweise mangelhafte Übereinstimmung zwischen Zielgruppen- und Angebotsprofil 
festgestellt. Auf diesem Hintergrund erstaunt es nicht, dass den Themen Vermittlung und Assessment sowie Case Management auch in der Sozialen Arbeit eine zunehmende Bedeutung zukommt.

Diese vier kurz beschriebenen Gestaltungsfragen bei Versorgungssystemen stellen spezifische Anforderungen an die aufgabenbezogene und strukturelle Passung der leistungserbringenden Organisationen innerhalb von Versorgungssystemen einerseits und an die politischen Akteure andererseits. Diese setzen in der Regel die Rahmenbedingungen für die Planung und Gestaltung von Versorgungsnetzwerken oder planen und steuern die Systeme mit den Fachstellen ihrer Verwaltungen selber.

Open Access Dieses Kapitel wird unter der Creative Commons Namensnennung 4.0 International Lizenz (http://creativecommons.org/licenses/by/4.0/deed.de) veröffentlicht, welche die Nutzung, Vervielfältigung, Bearbeitung, Verbreitung und Wiedergabe in jeglichem Medium und Format erlaubt, sofern Sie den/die ursprünglichen Autor(en) und die Quelle ordnungsgemäß nennen, einen Link zur Creative Commons Lizenz beifügen und angeben, ob Änderungen vorgenommen wurden.

Die in diesem Kapitel enthaltenen Bilder und sonstiges Drittmaterial unterliegen ebenfalls der genannten Creative Commons Lizenz, sofern sich aus der Abbildungslegende nichts anderes ergibt. Sofern das betreffende Material nicht unter der genannten Creative Commons Lizenz steht und die betreffende Handlung nicht nach gesetzlichen Vorschriften erlaubt ist, ist für die oben aufgeführten Weiterverwendungen des Materials die Einwilligung des jeweiligen Rechteinhabers einzuholen.

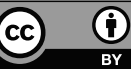

